\title{
APC-derived cytokines and T cell polarization in autoimmune inflammation
}

\author{
Ilona Gutcher and Burkhard Becher
}

Neuroimmunology Unit, Neurology Clinic, University of Zurich, Zurich, Switzerland.

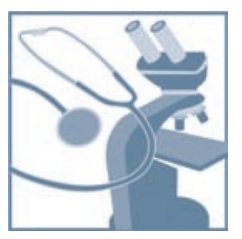

T cell-mediated autoimmune diseases such as multiple sclerosis and rheumatoid arthritis are driven by autoaggressive Th cells. The pathogenicity of such Th cells has, in the past, been considered to be dictated by their cytokine polarization profile. The polarization of such effector $T$ cells relies critically upon the actions of cytokines secreted by APCs. While Th1 polarization has long been associated with the pathogenesis of autoimmune diseases, recent data obtained in gene-targeted mice and the discovery of Th17 cell involvement in autoimmunity conflict with this hypothesis. In light of these recent developments, we discuss in this review the actions of APC-derived cytokines and their emerging roles in $\mathrm{T}$ cell polarization in the context of autoimmune inflammatory responses.

Tissue-specific autoimmune diseases, such as MS and RA, generally result from a loss of peripheral tolerance to self antigens, which leads to the inappropriate expansion of the self-reactive effector cell population and ensuing tissue-specific inflammation (reviewed in ref. 1). Although Th cells are widely believed to have a direct role in these diseases, APCs are key players during both the initiation and progression of the autoimmune response. APCs perform multifarious tasks in the peripheral immune compartment and, during an immune reaction, provide three signals that are required for the activation of antigen-specific T cells (Figure 1). The first signal involves the presentation of antigen on the surface of an MHC class II molecule, which facilitates $T$ cell recognition of the cognate antigen though the TCR. In order for antigen-specific T cells to become activated and this population to expand in number, a second signal must be generated through the interaction of adhesion and costimulatory molecules present on the APC, such as CD80 and CD86, with CD28 on the surface of T cells. The third signal is the secretion of cytokines by APCs, which directs the differentiation of activated antigen-specific lymphocytes into an effector $\mathrm{T}$ cell subtype. The creation of a particular cytokine environment by APCs during immunity is critical for the determination of the appropriate type of immune response, which can be either cell-mediated or humoral. Consistent with this, during an autoimmune reaction, the development of autoreactive $\mathrm{T}$ cells into pathogenic and destructive effector cells relies critically upon the secretion of soluble cytokines by APCs. Thus, the capacity of APC-derived cytokines to polarize T cells is of significant importance as it endows APCs with the potential to either promote or suppress the development of autoimmune disease. Here, we review our current knowledge of the various APC-derived cytokines that constitute signal 3 of an immune response, and in particular, we concentrate on their function in the context of autoimmunity.

Nonstandard abbreviations used: AIA, antigen-induced arthritis; CIA, collageninduced arthritis; CII, collagen type II; CSF, cerebrospinal fluid; GATA3, GATA-binding protein 3 ; IFN- $\gamma$ R, IFN- $\gamma$ receptor; MOG, myelin oligodendrocyte glycoprotein; T-bet, T-box expressed in T cells.

Conflict of interest: The authors have declared that no conflict of interest exists. Citation for this article: J. Clin. Invest. 117:1119-1127 (2007). doi:10.1172/JCI31720.

\section{The Th1/Th2 paradigm of autoimmunity}

In 1986 Mosmann et al. initially proposed a model whereby $\mathrm{CD}^{+} \mathrm{T}$ cells are subdivided into two independent subsets with distinct effector functions $(2,3)$. Their hypothesis suggested that Th cells can be segregated into Th1 and Th2 subsets on the basis of cytokine expression and bioactivities as well as helper function. Those and further experiments demonstrated that Th1 cells secrete predominantly IL-2, IL-3, TNF- $\alpha$, and most notably IFN- $\gamma$ and control cell-mediated functions such as the activation of macrophages, while the secretion of IL- 4 , IL-5, and IL- 13 by Th2 cells leads to the stimulation of humoral immunity by aiding $B$ cell activation and class switching (3). Interestingly, the cytokines of a particular Th subtype are able to further promote the expansion of that subtype population while simultaneously inhibiting the development of the other subset. This allows each Th subset to produce characteristic cytokines that in turn provoke the development of a distinctive effector function specific for that immunogen. Thus, while Th1 cells induce proinflammatory responses, such as delayed-type hypersensitivity, and eliminate intracellular infections, Th2 cells mediate allergic reactions and anti-helminth responses (1).

The differentiation of lymphocytes into Th1 or Th2 cells is absolutely dependent upon the production of cytokines by APCs. For the purpose of this review, the term APCs will refer to macrophages and DCs, although B cells are also capable of presenting antigen. APCs are innate immune cells that are activated by microbial components via pattern-recognition receptors such as TLRs. Such triggering stimulates the secretion of a precise set of cytokines that ultimately results in a specific reaction against that pathogen by the induction of a Th1 or Th2 cell response. APCs thus bridge the innate and adaptive arms of immunity. It has been demonstrated that the APC-derived proteins IL-12 and IL-18 are key players in the development of a Th1 cell response and that they synergize with one another by upregulating their reciprocal receptors on the surface of Th1 cells $(4,5)$. Alternatively, Th2 cell differentiation seems to be promoted solely by $\mathrm{T}$ cell-derived cytokines, in particular IL-4, subsequent to interaction with APCs.

Until recently, the development of autoimmune diseases was also conceptualized as being governed by the Th1/Th2 subset model, whereby it was widely held that Th1 cells form the patho- 

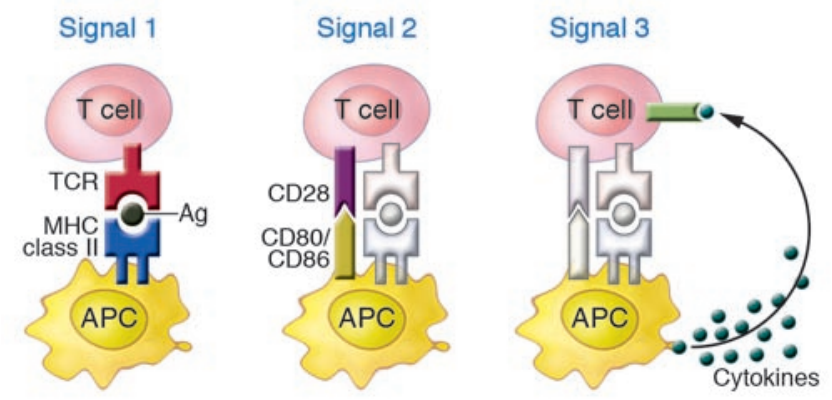

Figure 1

Within the immune synapse formed between APCs and T cells, three signals are required for antigen-specific $T$ cell activation. Signal $1 \mathrm{com}-$ prises the presentation of antigen peptide, in the context of MHC class II molecules, which is recognized by the antigen-specific TCR. Signal 2 involves the stabilization of the synapse through adhesion molecules and the generation of signals via costimulatory molecules present on the surface of APCs and T cells. CD80/CD86 on APCs interact with their receptor, CD28, on T cells to generate activatory signals, while interaction with cytotoxic T lymphocyte-associated protein 4 (CTLA4) generates inhibitory signals (not shown). Signal 3 is produced by the secretion of cytokines by APCs, which signal via cytokine receptors on T cells in order to polarize them toward an effector phenotype. Ag, antigen.

genic $\mathrm{T}$ cell subset in the context of tissue-specific $\mathrm{T}$ cell-driven autoimmune diseases, while Th2 cells are thought to mediate allergic responses and asthma. Conversely Th2 cells were reported to exert beneficial function during tissue-directed autoimmune disease pathogenesis (6). With regard to autoimmune inflammation of the CNS or joints, this paradigm was proposed on the basis of several observations: tissue-invading Th cells normally express IFN- $\gamma$ (7); Th1-inducing cytokines are present in the inflammatory lesion and often correlate with disease severity (8); and EAE, the animal model for MS, can be induced by the adoptive transfer of encephalitogenic Th1 cells $(9,10)$. Indeed, treatment of mice with Th1-inducing cytokines mostly results in aggravation of autoimmune diseases, such as EAE and collagen-induced arthritis (CIA), the animal model for RA (for a list of reports in support of the Th1/2 autoimmunity paradigm, see Evidence for Th1 pathogenesis in autoimmunity). However, studies involving the generation and immunization of mice deficient in Th1 cytokines such as IFN- $\gamma$ and TNF- $\alpha$ demonstrated that they were not, contrary to expectations, protected from autoimmune disease. Disease progression and severity of both EAE and CIA in TNF- $\alpha$-deficient mice was identical to that in WT mice, while IFN- $\gamma$ - and IFN- $\gamma$ receptor-deficient (IFN- $\gamma \mathrm{R}$-deficient) mice were hypersusceptible to disease $(11,12)$. Loss of IFN- $\gamma$ even causes disease-resistant mouse strains to become highly susceptible to EAE (13). In addition, deletion of Th2 cytokines generally did not affect the progression of autoimmune disease (14). The disease-exacerbating effects of Th1 cytokine deficiency thus presents a dichotomy, as it suggests protective effects for Th1 cytokines. Despite these contradictory results, autoimmunity has persisted to be defined by the Th1/Th2 paradigm mostly due to data describing autoimmune disease in mice deficient either in the transcription factor for IFN- $\gamma$ or in Th1-inducing cytokines. T-box expressed in T cells (T-bet) is the key transcription factor for Th1 cell development and IFN- $\gamma$ production, and mice deficient in T-bet do not develop Th1 cells or produce IFN- $\gamma(15,16)$. Consistent with a role for Th1 cells and
IFN- $\gamma$ in autoimmunity, T-bet-deficient mice are resistant to the induction of several experimental autoimmune diseases, such as EAE, inflammatory bowel disease (IBS), and SLE (17-20). Protection from disease correlated with a skewing in the ratio of the Th1/Th2 response toward a more dominant Th2 environment that included significant upregulation of the Th2 cytokines IL-4, IL-5, and IL-10. Conversely, inhibition of GATA-binding protein 3 (GATA-3) expression reduces Th2 cytokine expression, thereby decreasing allergic airway inflammation and hyperresponsiveness (21). Such an imbalance in the Th1/Th2-inducing transcription factors has also been observed during human disease, as relapsing-remitting MS patients have an increased expression of T-bet in peripheral blood leukocytes during relapse (22). On the other hand, increased gene expression of GATA3 and reduced expression of T-bet have been demonstrated in the airways of patients with asthma $(23,24)$. In light of the above data (see Evidence for Th1 pathogenesis in autoimmunity), immune-modifying strategies for the treatment of autoimmune inflammation have focused on the concept of immune deviation (reviewed in ref. 25), whereby autoaggressive Th1 cells are deviated toward a Th2-polarized phenotype. Thus, while the Th1/Th2 paradigm of autoimmunity has been bombarded with conflicting data, the fact that T-bet-deficient mice, as well as mice deficient in APC-derived, Th1-inducing cytokines, are resistant to the induction of antigen-driven autoimmune diseases has held this paradigm firmly in place. We will now discuss the role of APC-derived cytokines and the paradigm shift regarding their function in autoimmune inflammation that was finally initiated by a number of key findings.

\section{IL-12 and IL-18: major commanders of Th1 polarization}

APC-derived cytokines constitute signal 3 of $\mathrm{T}$ cell activation, and critically, they determine the outcome of $\mathrm{T}$ cell differentiation, indicating that they play a significant role in governing immunity. Mice deficient in Th1-inducing cytokines, such as IL-12 and IL-18, have been shown to be completely resistant to EAE and demonstrated reduced incidence and severity during CIA, which was again concomitant with reduced Th1 cell development. In addition, the treatment of mice with neutralizing Abs against IL-12p40 effectively prevents disease induction and suppresses EAE in adoptive transfer recipients (26). In CIA, IL-12 Ab treatment reduced the severity of disease but not the incidence, an effect that was associated with reduced IFN- $\gamma$ production by restimulated lymph node cells; decreased spontaneous TNF, IL-6, and IL-10 production by synovial cells ex vivo; and a reduction in total anti-collagen type II (anti-CII) Abs (27). Notably however, administration of antiIL-12 Abs showed efficacy in IFN- $\gamma$-deficient mice, demonstrating IFN- $\gamma$-independent actions (28). The treatment of mice with recombinant IL-12 is capable of exacerbating EAE and CIA, which is again thought to result from enhanced activation of IFN- $\gamma-$ producing T cells (29). Of significant importance, roles for Th1inducing cytokines can also be determined from studies of human disease. In MS, patients demonstrate increased levels of IL-12 in serum (30), cerebrospinal fluid (CSF) (31), MS lesions, and PBMCs when compared with controls (32). It has also been shown that augmented IL-12 expression is representative of the degree of disability, as IL-12p40 mRNA is elevated in MS patients during the development of active lesions and immediately prior to relapses during relapsing-remitting MS (33). Likewise, IL-12 is present in the inflamed synovium of RA patients both in synovium-infiltrating macrophages and synovial lining cells $(34,35)$. Taken together, 


\section{Evidence for Th1 pathogenesis in autoimmunity}

\section{Th1 cytokines in autoimmune disease}

$\uparrow$ IL-12 demonstrated in plasma, CSF, PBMCs, and plaques of MS patients (30-33, 98-100)

Higher percentage of IL-12R $\beta 1$ - and IL-12R $\beta 2$-expressing T cells in CSF compared with blood of MS patients (101)

$\uparrow$ IFN- $\gamma$ and IL-12 in synovial fluid monocytes compared with PBMCs of both RA patients and healthy controls (102)

Strong expression of IL-12p40 and IL-12p70 in synovial tissue of RA patients (34)

$\uparrow$ IL-18 expression in serum, PBMCs, and demyelinating lesions of MS patients (103-105)

$\uparrow$ IL-18 expression in synovial tissues of RA patients $(106,107)$

Synovial IL-18 expression correlates directly with RA disease activity and response to disease therapy (108)

$\uparrow$ Serum levels of IFN- $\gamma$ in RR-MS patients prior to onset of relapse (109)

Significant positive correlation between IFN- $\gamma$-producing PBMCs and MS disability (110)

$\uparrow$ IL-12, IL-2, IFN- $\gamma$, TNF- $\alpha$, IL-1 $\beta$, and IL- 6 in PBMCs of patients with acute and relapsing MS (100)

\section{Th1 cytokines in experimental autoimmune disease}

Anti-IL-12p40 Abs reduced CIA severity but not incidence associated with $\downarrow$ IFN- $\gamma$ levels (27)

Anti-IL-12p40 Ab suppresses EAE in adoptive transfer recipients (26)

IL-12p40-deficient mice are resistant to MBP-induced EAE (28)

IL-12 treatment increases development of IFN- $\gamma$-producing CD4 $4^{+}$T cells and thus increases the severity of CIA (29)

Anti-IL-18 Abs significantly $\downarrow$ IFN- $\gamma$ production and disease development during MBP-induced EAE in rats (111)

IL-18-deficient mice are resistant to $\mathrm{MOG}_{35-55}$-induced EAE due to decreased IFN- $\gamma$ production (71)

$\downarrow$ Incidence and severity of CIA in IL-18-deficient mice (72)

$\downarrow$ CIA severity in mice treated with anti-IL-18 Ab or rhIL-18BP (112)

CNS-infiltrating cells of EAE mice stained for IFN- $\gamma$ and IL-2 (8)

$\mathrm{CD}^{+} \mathrm{T}$ cells infiltrating the CNS of acute EAE mice produced the Th1 cytokines IL-2 and IFN- $\gamma$ (7)

T-bet-deficient mice completely failed to generate Th1 cells and thus are resistant to $\mathrm{MOG}_{35-55}$-induced EAE (17)

MBP, myelin basic protein; rhIL-18BP, recombinant human IL-18 binding protein; RR-MS, relapsing-remitting MS. $\uparrow$, increased; $\downarrow$, decrease(d).

data from human autoimmune disease as well as the animal models both point toward a highly relevant role for Th1-inducing conditions and IFN- $\gamma$ production in autoimmune pathogenesis (see Evidence for Th1 pathogenesis in autoimmunity).

\section{A case of mistaken identity?}

IL-12 is a heterodimeric cytokine of approximately $70 \mathrm{kDa}$ consisting of heavy (i.e., p40) and light (i.e., p35) subunits, which are covalently linked through disulfide bonds. While p40 appears to be produced in abundance in IL-12-expressing cells, p35 is ubiquitously and constitutively expressed only at low levels, and it requires p40 coexpression for secretion of the biologically active cytokine from the cell. The wealth of data pertaining to IL-12's role in autoimmunity originated from studies using mice deficient in the p40 subunit of IL-12 as well as the administration of Abs that turned out to be specific for IL-12p40, both of which abolish expression and/or the function of IL-12. However, a series of reports has led to a revised concept of IL-12 function in autoimmune disease, starting in 2000 with further evidence that the p40 subunit is able to dimerize with subunits other than p35 (36). While it was previously known that $\mathrm{p} 40$ could homodimerize to form $\mathrm{p} 80$ with puta- tive IL-12 antagonistic activities (37), Oppmann and colleagues identified an IL-6/IL-12-related subunit, p19, with which p40 heterodimerizes to form the cytokine IL-23 (36). Upon ubiquitous transgenic expression of $\mathrm{p} 19$ in mice, Wiekowski et al. showed that p19 has biological properties similar to those of IL-6, G-CSF, and IL-12, which resulted in the induction of multiorgan inflammation in these animals (38). In 2002, we were able to demonstrate that immunized mice deficient in IL-12p35 were surprisingly not resistant to myelin oligodendrocyte glycoprotein-induced (MOGinduced) EAE but actually had increased clinical severity, a finding that was reproduced by others $(39,40)$. Interestingly, the conflicting phenotypes were accompanied by a decrease in Th1 immunity in both disease-susceptible p35-deficient mice and disease-resistant $\mathrm{p} 40$-deficient mice (39). In addition, mice lacking the p40binding receptor subunit of IL-12 - IL-12R $\beta 1$ - were also resistant to EAE, but the p35-binding subunit, IL-12R $\beta 2$, was shown to be unnecessary (41). The discordant effects of IL-12p40 and IL-12p35 clearly suggest an indispensable role for $\mathrm{p} 40$ but not for IL-12 in experimental disease. The incompatibility of the p40 and p35 phenotypes seemed likely to result from the promiscuity of the 40 subunit, whereby resistance of $\mathrm{p} 40$-deficient mice resulted from 


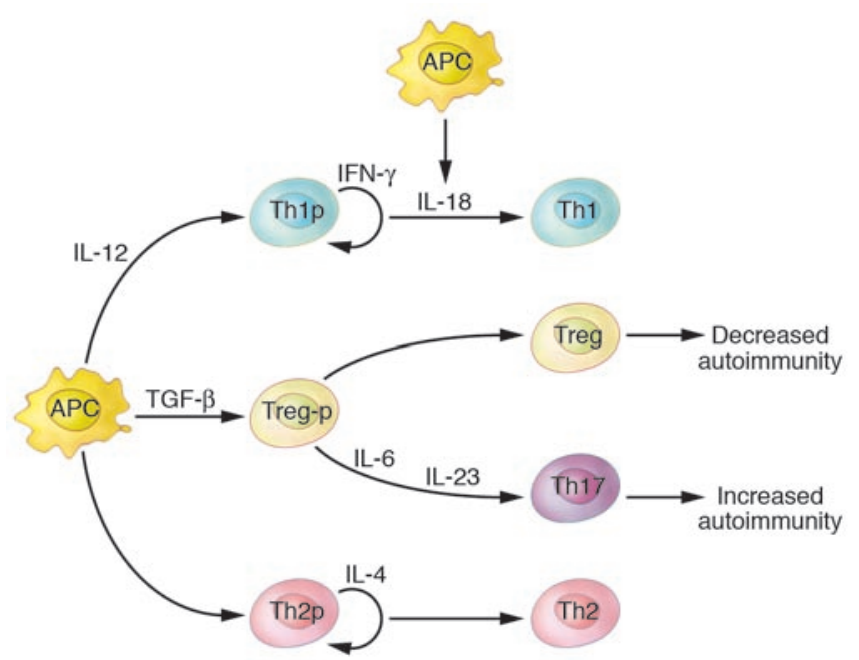

Figure 2

APC-derived cytokines guide the differentiation of naive T cells into an effector T cell subtype. Secretion of IL-12, in synergy with IL-18, leads to the generation of Th1 cells. Initial IL-12 production directs the upregulation of IL-18R and IL-12R $\beta 2$ expression on the surface of Th1 precursor (Th1p) cells that allows IL-18 to aid IL-12 in Th1 polarization. TGF- $\beta$ secretion can polarize naive cells toward a regulatory phenotype or an autoaggressive phenotype, depending on the cytokine environment: secretion of TGF- $\beta$ alone by APCs supports Treg formation (which counteracts autoimmune inflammation) from Treg precursor cells (Treg-p cells). However, the additional presence of IL-6 results in the production of Th17 cells, which are now considered to be the pathogenic $T$ cell population during autoimmunity. The pathogenic, APC-derived cytokine IL-23 is critical for the maintenance and survival of these autoreactive Th17 cells. The interaction of APCs and Th2 precursor (Th2p) cells in the absence of IL-12 and IL-18 induces the production of the Th2 cytokine IL-4 by T cells, which acts in an autocrine fashion to polarize committed Th2 cells.

the lack of IL-23 and not IL-12. This was later confirmed in mice deficient in the IL-23 subunit p19, and therefore in IL-23 alone, which, like p40-deficient mice, were protected from both EAE and CIA $(42,43)$. In contrast to 440 deficiency, p19 deficiency does not affect the development of Th1 cells and IFN- $\gamma$ production, which provides further evidence against a critical pathogenic role for Th1 cells in autoimmunity.

IL-23 is secreted by APCs and signals via the high-affinity receptor complex IL-23R, which is made up of the p40-binding IL-12R $\beta 1$ subunit and the signaling IL-23R subunit. IL-23R is present on the surface of various cells of the immune system, including activated/ memory T cells, NK cells, DCs, monocytes, and macrophages. In contrast to IL-12R, IL-23R is highly expressed on murine memory $\mathrm{CD}^{+} \mathrm{T}$ cells and is expressed at low levels on naive T cells, permitting unique effects of IL-23 on this cell type. As demonstrated in p19-/- mice, IL-23 does not affect Th1 polarization, as evidenced by an intact IFN- $\gamma$ response (44). In contrast, IL-23 is one of the factors essential for the expansion and survival of another, newly defined Th cell subset that secretes IL-17 and is consequently known as the Th17 subset.

\section{Th17 cells}

After the discovery of a new Th cell subset by Langrish et al. (44), a large number of articles in short succession were dedicated to the regulation of IL-17 expression by such Th17 cells. IL-17 (also known as IL-17A) is a proinflammatory cytokine that induces the expression of IL-1, IL-6, G-CSF, as well as chemokines, by cells such as fibroblasts, stromal cells, and endothelial cells (45-51). It is secreted by $\mathrm{CD}^{+}$Th17 cells, which characteristically also produce IL-17F (another IL-17 family member, with the closest sequence identity to IL-17A), IL-6, TNF- $\alpha$ (44, 52), and IL-22 (53-55). Indeed, this Th17 subset is now considered to constitute the critical population responsible for mediating pathogenesis during autoimmunity. The expression of IL-17 has been associated with numerous human autoimmune diseases, such as RA $(56,57)$ and MS $(58,59)$, and its inhibition or deletion in the corresponding animal models has provided a varying degree of protection (44, 60-63). A delicate balance of cytokines promotes the differentiation of naive $\mathrm{CD}^{+} \mathrm{T}$ cells into either polarized Th17 cells or autoimmunity-suppressing Tregs. While TGF- $\beta$ stimulates polarization of $\mathrm{CD}^{+} \mathrm{T}$ cells into Tregs in the absence of IL- 6 in vitro, the additional presence of IL- 6 evidently shifts the balance toward the proinflammatory Th17 phenotype, a process that is amplified by IL-1 $\beta$ and is negatively regulated by Th1 and Th2 cytokines (43, 63-65) (Figure 2). Both in vitro and in vivo differentiation of Th17 cells require induction of the transcription factor retinoic acidrelated orphan receptor- $\gamma \mathrm{t}(\mathrm{ROR} \gamma \mathrm{t})$, which is characteristic of this new $T$ cell subset (66). In light of this emerging $T$ cell subset, some of the previously observed discrepancies in the Th1/Th2 paradigm of autoimmunity could be explained, especially with regard to the conflicting data obtained for IL-12p40- and IL-12p35-deficient mice. Lymphocytes obtained from EAE-resistant IL-12p40-/- mice demonstrate defects in both Th1 and Th17 responses, while cells from hypersusceptible IL-12p35/- mice, which still express IL-23, lack Th1 development but have an elevated frequency of Th17 cells $(67,68)$. Consistent with these findings, EAE-resistant IL-23p19-/mice show normal Th1 responses but have significantly reduced IL-17 production (44). Adoptive transfer experiments showed that encephalitogenic $\mathrm{CD}^{+} \mathrm{T}$ cells that had been treated in vitro with antigen and IL-23, but not IL-12, could induce EAE in recipient mice (44). In accordance with a role for IL-23 in the expansion and survival of Th17 cells, IL-23 has been demonstrated to be required during the effector phase of $\operatorname{EAE}(42,69)$ (Figure 2). The generation of bone marrow chimeric mice, in which $\mathrm{p} 40$ was expressed either in the CNS or the peripheral immune system, showed that $\mathrm{p} 40$ is not absolutely required for the infiltration of inflammatory cells into the CNS but that $\mathrm{p} 40$ produced by cells resident to the CNS is essential for maintaining encephalitogenicity of T cell migration into the CNS (69). Furthermore, Cua et al. showed that injection of IL-23 into the CNS of $\mathrm{p}^{19-/-}$ mice abrogated resistance of these animals to EAE (42). They suggest that the role of IL-23 in promoting encephalitogenicity results from its activation of macrophages present in the inflamed CNS to produce proinflammatory cytokines such as IL-1 and TNF. This points to an important role for IL-23 in the regulation of myeloid cells in organ-specific autoimmune diseases. However, the precise action of IL-23 in autoimmunity apart from its direct effect on Th cells still needs to be elucidated.

The above findings with regard to IL-12 and IL-23 in the context of CNS autoimmune inflammation were also reproduced in the animal model of autoimmune joint inflammation (i.e., CIA) $(43,70)$. Mice lacking IL-23 are resistant to disease induction and show no pathology upon histological examination of the joints. IL-12p35/- mice, however, have an enhanced CIA severity and a delayed disease recovery in comparison with WT mice, although 


\section{Evidence for Th17 pathogenesis in autoimmunity}

IL-12p35 $1 /-$ mice are susceptible to $\operatorname{EAE}(39,40)$

IL-23 ${ }^{-/-}$mice are resistant to EAE (42)

IL-12 $35^{-/-}$mice are susceptible to CIA, while IL-12p40/- and IL-23p $19^{-/-}$mice are protected from CIA (43)

IL-18 $/-$ mice are susceptible to EAE, EAU, and AIA (79-81)

IFN- $\gamma^{-/-}$mice are hypersusceptible to $\operatorname{EAE}(11,13)$

IFN- $\gamma^{-/-}$and IFN- $\gamma \mathrm{R}$-deficient mice are hypersusceptible to CIA (113-115)

TNF- $\alpha^{-/-}$mice are not resistant to EAE (12)

IL-4 $/-$ mice are not hypersusceptible to EAE (14)

IL-23 induces a population of encephalitogenic CD4 $4^{+} \mathrm{T}$ cells called Th17 cells (44)

IL-17 block or deficiency reduces susceptibility to EAE and CIA $(44,60,61,63)$

IL-1 is necessary for the generation of Th17 cells (92)

IL-18R is necessary for the generation of Th17 cells (79)

IL-17 mRNA is augmented in blood and CSF mononuclear cells of MS patients (58)

IL-17 is produced by the rheumatoid synovium (56)

EAU, experimental autoimmune uveoretinitis.

there is no difference at the histopathological level (43). IL-23 or IL-12 deficiency did not affect arthritis development at the level of CII-specific T cell proliferation; however, gene expression analysis demonstrated a decrease in IFN- $\gamma$ and an increase in IL- $1 \beta$, IL-6, IL-17, TNF, and nitric oxide synthase 2 in the absence of IL-12. Despite the increased levels of IFN- $\gamma$, there was no difference in the expression of IFN- $\gamma$-associated chemokines in the joints of IL-12-/- mice. Therefore, IL-23 and not IL-12 is required for mediating the pathogenesis of autoimmune joint inflammation, which is subsequently, to some extent, modulated by the IL-12/IFN- $\gamma$ pathway (for a list of reports rejecting the Th1/Th2 paradigm, see Evidence for Th17 pathogenesis in autoimmunity).

\section{IL-18: another Th1 inducer lost to autoimmune pathology}

The evidence discussed above clearly argues against a role for IFN- $\gamma$ secreting Th1 cells in the pathogenesis of autoimmune disease and suggests a more regulatory role for this subset (Evidence for Th17 pathogenesis in autoimmunity). However, given the known actions of IL-18, as well as the previously discussed role of T-bet, this paradigm cannot be completely dismissed. IL-18 is an $18-\mathrm{kDa}$ proinflammatory cytokine and a member of the IL-1 superfamily of cytokines, although its activities are more similar to those of IL-12. It is, like IL-12, expressed by macrophages and DCs but also by epithelial cells, keratinocytes, and other stromal cells. It signals through the IL-18R, a heterodimeric complex of an IL-18R $\alpha$-binding subunit and an IL-18R $\beta$ signaling subunit (also termed IL-1RAcPL or IL-1R7). The extracellular portion of IL-18R $\alpha$ is responsible for IL-18 binding. However, this requires the presence of the receptor $\beta$ subunit, which comprises the high-affinity receptor complex. IL-18R is expressed by a variety of immune cells, including lymphocytes, NK cells, macrophages, and neutrophils. As a result, IL-18 has pleiotrophic effects, although it is best known for its promotion of Th1 differentiation and IFN- $\gamma$ production through its synergistic activities with IL-12 (Figure 2). It can also independently stimulate the cytotoxic activities of NK cells. It has previously been shown that IL-18 $/-$ mice are protected from $\mathrm{MOG}_{35-55}$-induced EAE and are unable to mount autoreactive Th1 responses (71). The defective Th1 cell response was suggested to result at least in part from abrogated IFN- $\gamma$ production by NK cells, as transfer of NK cells from RAG-deficient, but not from IFN- $\gamma^{-/}$, mice was able to rescue the susceptibility of these mice to EAE. In CIA, IL-18 deficiency reduced disease severity and incidence, characterized by decreased TNF- $\alpha$ in serum and spleen cultures and suppressed CII-specific Th1 responses (72). Administration of recombinant IL-18 to mice with CIA significantly enhances disease, with increased synovial hyperplasia, cellular infiltration, and cartilage erosion being observed (73). The effects of IL-18 are of a proinflammatory nature, as a significant increase in the production of IFN- $\beta$, TNF- $\alpha$, and IL-6 occurred in these mice (74). The effects of IL-18 on bone and cartilage remain controversial, as both stimulatory and inhibitory effects on osteoclast formation have been reported (75-77). In contrast, a role for Th17 cells and Th17-mediated osteoclastogenesis in inducing autoimmune arthritis-related bone destruction has recently been established (78).

The discovery that IL-12 is superfluous during autoimmune pathogenesis while IL-18 is an absolute necessity raised the question of whether there is redundancy in the system whereby IL-18 can function in the absence of IL-12 to induce autoimmunity. We recently were able to show that this was not the case, as mice deficient in both IL-12p35 and IL-18 were still fully susceptible to MOG peptide-induced EAE (79). On further analysis, and in contrast to the previous report, we discovered that IL-18-deficient mice were fully susceptible to EAE. Furthermore, IL-18 $18^{-/}$mice produced $\mathrm{T}$ cell responses and IFN- $\gamma$ levels that were identical to those of WT mice. Despite a clear contradiction between our findings and those of Shi et al. $(71,79)$, this was not the first time that a redundant role for IL-18 has been described in a model of autoimmune disease. Santos et al. used a model of autoimmune arthritis 
known an antigen-induced arthritis (AIA) that is induced with methylated BSA in CFA and, in contrast to CIA, is dependent on cell-mediated immunity only (80). Applying this model, they were able to show that IL-18 is not required for AIA, nor is it necessary as a Th1 response cofactor and inflammatory cytokine (80). The conflicting results described for IL-18 in EAE and AIA models have been postulated to result from several possible causes. Differences in backcrossing or the health status of the mouse are likely to be the main reasons, while, in the arthritis model, variability may have resulted from the divergent pathogenic mechanisms of these models. However, genetic background of the mice may also play a role. While IL-18 ${ }^{-/}$mice were backcrossed to the arthritis-susceptible DBA/ 1 background for CIA induction, AIA experiments were performed in IL-18 1 - mice backcrossed onto the C56BL/6 background. Speaking against this hypothesis is the demonstration that IL-18-deficient mice on a DBA/1 background are not protected from ocular experimental autoimmune uveoretinitis (EAU), an autoimmune disease targeting photoreceptor antigens of the eye (81). It would nevertheless be of significant interest to determine the susceptibility of IL-18 $1 /$ mice to CIA that are not on the DBA background.

We felt that studying the susceptibility of IL-18R $\alpha^{-/-}$mice to EAE could potentially shed light on the conflicting data discussed above. In contrast to the emerging nonpathogenic role for IL-18 in autoimmunity, which is in line with the changing Th1/Th2 paradigm, we discovered that IL-18R $\alpha$ deletion induces resistance to EAE development (79). The administration of anti-IL-18R $\alpha$ Abs to IL-18 $/-$ mice abrogated disease development both before and after disease onset, leading us to conclude that an alternative ligand exists that is capable of binding to and signaling through IL-18R $\alpha$ to induce autoimmunity. Finally, IL-18R $\alpha^{-/-}$mice failed to generate and/or sustain encephalitogenic Th17 cells. In this context, we discovered that IL-18R $\alpha$ engagement on accessory cells, and not lymphocytes, results in defective IL-12/23p40 production.

In light of the fact that the affinity of IL-18 to IL-18R $\alpha$ is weak (82) and that there are still a number of orphan ligands in the IL-1 superfamily, we propose that an unknown alternative ligand binds to IL- $18 \mathrm{R} \alpha$ and a so-far-unidentified signaling subunit expressed on APCs. Engagement of the IL-18R $\alpha$ by this ligand supports the production of IL-23 necessary for the consequent expansion of Th17 cells.

Strengthening the findings that IL-18R $\alpha$ is capable of interacting with a ligand other than IL-18, Lewis and Dinarello recently showed opposite responses in the rejection of $\mathrm{IL}_{-18^{-/}}$and IL-18R $\alpha^{-/-}$pancreatic islets (83), which led the authors to propose the existence of an IL-18-independent inhibitory pathway that converges with the proinflammatory IL-18 signaling pathway at the IL-18R. Furthermore, they suggest the involvement of IL-1F7, a member of the IL-1 family that binds IL-18 binding protein (IL-18BP) and IL-18R $\alpha$, thereby inhibiting IL-18 activity.

Taken together, the data indicate that the polarization of Th17 cells clearly correlates with pathogenicity, while APC-derived cytokines have a critical role in polarizing $\mathrm{T}$ cells toward this autoimmunogenic phenotype (84). Thus, it would be of interest to reassess the role of the Th17-inducing cytokines IL-1, IL-6, and TGF- $\beta$ in autoimmunity. It has long been known that both IL-1 and IL- 6 are present in the synovial fluid of RA patients $(85,86)$, whereas in MS, IL-6 was upregulated in the plasma and CSF of MS patients, although no correlation between IL-6 levels and disease activity could be made (87). Correspondingly, IL-6-/- mice are protected from EAE and have delayed onset and reduced severity of CIA $(88,89)$. IL-1/- mice are resistant to CIA, and IL-1 $\mathrm{R}^{-/-}$mice are protected from $\operatorname{EAE}(90,91)$. However, as IL-17 itself stimulates the production of both these molecules, this begs the question whether these in vitro Th17-inducing cytokines act upstream or downstream of IL-17. With regard to IL-1, Sutton et al. have provided evidence pointing to an upstream role for IL-1 (92). They showed that EAE resistance of IL-1R $\mathrm{R}^{-/-}$mice results from deficient IL-17 production and that the adoptive transfer of encephalitogenic T cells can induce EAE in these mice (92). Furthermore, the spontaneous development of autoimmune arthritis in IL-1R antagonist-deficient (IL-1Ra-deficient) mice was completely prevented in mice on an IL-17-deficient background (93). Together, these data suggest that IL-1 is required for EAE during the development but not during the effector stages through its induction of Th17 cells. With regard to IL-6, Hirota et al. have demonstrated that the spontaneous occurrence of autoimmune arthritis in SKG mice results from Th17 cell development, but only in the presence of IL-6 (94). Therefore, IL-6 also appears to act upstream of Th17 cells. Adding complexity to the Th-17 pathway is the recent discovery that IL-25, also known as IL-17E and a member of the IL-17 family of cytokines, can regulate Th17 function in autoimmune inflammation (95). IL-25/- mice are hypersusceptible to EAE as a result of increased IL-23 expression and a subsequent increase in the number of Th17 cells. The effects of IL-25 were mediated through elevation of IL-13, which in turn inhibits IL-23, IL-1 $\beta$, and IL-6 expression in activated DCs (95).

\section{Concluding remarks}

While the Th1/2 paradigm has for more than a decade served to conceptually encompass the role and function of Th cells in autoimmune disease, it has not withstood the recent developments in molecular biological techniques that have permitted the ultimate testing of the hypothesis using gene-targeted animals. Initial findings that Th1-deficient mice are still susceptible or even hypersusceptible to autoimmune disease proved conflicting until the discovery that APC-derived, Th1-polarizing cytokines are also redundant during autoimmune pathogenesis. The remaining inconsistency preventing the complete disintegration of the Th1/ Th2 paradigm is the evidence that mice deficient in the Th1 transcription factor T-bet are resistant to EAE. However, it is feasible that resistance to autoimmune disease in T-bet-deficient systems is the result of mechanisms other than the inability of $\mathrm{CD}^{+} \mathrm{T}$ cells to differentiate into Th1 cells: T-bet has been shown to be essential for optimal selectin ligand expression in Th1 cells and their subsequent trafficking $(96,97)$; significantly upregulated levels of IL-10 have been demonstrated in T-bet ${ }^{-/-}$mice, indicating a possible increase in and effect of immunoregulatory Tregs (18); a comparison of WT CD $4{ }^{+} \mathrm{CD} 25^{+} \mathrm{T}$ cells and T-bet ${ }^{-/-} \mathrm{CD} 4^{+} \mathrm{CD} 25^{+}$ cells showed that $\mathrm{T}^{-}$bet ${ }^{-/}$cells had an enhanced ability to suppress proliferation of WT CD $4^{+} \mathrm{CD} 25^{-}$and $\mathrm{T}$-bet ${ }^{-/-} \mathrm{CD} 4^{+} \mathrm{CD} 25^{-}$effector T cells (17). Furthermore, it has been suggested that T-bet may play a role in IL-23 responsiveness during $\mathrm{T}$ cell differentiation (68).

Despite the somewhat conclusive evidence indicating a pathogenic role for Th17 cells, anti-IL-17 Ab treatment $(44,63)$ or IL-17 deficiency (60) in mice does not yield complete resistance to autoimmune disease in contrast to deficiency in IL-23 $(42,43)$ or factors promoting IL-23 such as IL-18R $\alpha$ (79). This suggests a more complex network downstream of IL-23 signaling, involving factors in addition to IL-17. It has recently been demonstrated that Th17 
cells concomitantly produce IL-22, whose expression is controlled by the same IL-17-promoting cytokines $(53,54)$. IL-22 has been demonstrated to have a pathogenic role in psoriasis (55), but it remains to be shown whether this cytokine is important in other autoimmune inflammatory diseases. Therefore, there are likely other as-yet-unidentified factors either upregulated or suppressed by IL-23. Last, the dominant role of IL-23 may not only be related to its impact on pathogenic T cells. Accessory cells such as macrophages and DCs also express the receptor of IL-23, and its impact on disease is yet to be fully elucidated. The evidence against the Th1/Th2 paradigm of autoimmunity and in support of IL-23/ IL-17-mediated mechanisms is overwhelming and demonstrates an indispensable role for APC-derived cytokines in promoting and/or suppressing autoimmune disease. The recent observations that IL-18 and IL-18R $\alpha$ produce different effects in autoimmune disease $(79,83)$ demonstrate the complexity of the system and suggest that in addition to unknown $\mathrm{T}$ cell factors, additional APCderived cytokines are secreted and necessary for $\mathrm{T}$ cell differentiation and autoimmune disease pathogenesis.

Address correspondence to: Burkhard Becher, Neuroimmunology Unit, Neurology Clinic, University of Zurich, Y44 J7 Winterthurerstrasse 190, Zurich 8057, Switzerland. Phone: 41-44-635-3703; Fax: 41-44-635-6883; E-mail: Burkhard.Becher@neuroimm.unizh.ch.
1. Moss, R.B., et al. 2004. Th1/Th2 cells in inflammatory disease states: therapeutic implications. Expert Opin. Biol. Ther. 4:1887-1896.

2. Mosmann, T.R., Cherwinski, H., Bond, M.W., Giedlin, M.A., and Coffman, R.L. 1986. Two types of murine helper T cell clone. I. Definition according to profiles of lymphokine activities and secreted proteins. J. Immunol. 136:2348-2357.

3. Coffman, R.L. 2006. Origins of the $\mathrm{T}(\mathrm{H}) 1-\mathrm{T}(\mathrm{H}) 2$ model: a personal perspective. Nat. Immunol. 7:539-541.

4. Yoshimoto, T., et al. 1998. IL-12 up-regulates IL-18 receptor expression on T cells, Th1 cells, and B cells: synergism with IL-18 for IFN-gamma production. J. Immunol. 161:3400-3407.

5. Chang, J.T., Segal, B.M., Nakanishi, K., Okamura, H., and Shevach, E.M. 2000. The costimulatory effect of IL-18 on the induction of antigen-specific IFN-gamma production by resting T cells is IL-12 dependent and is mediated by up-regulation of the IL-12 receptor beta2 subunit. Eur. J. Immunol. 30:1113-1119.

6. Racke, M.K., et al. 1994. Cytokine-induced immune deviation as a therapy for inflammatory autoimmune disease. J. Exp. Med. 180:1961-1966.

7. Renno, T., Krakowski, M., Piccirillo, C., Lin, J.Y., and Owens, T. 1995. TNF-alpha expression by resident microglia and infiltrating leukocytes in the central nervous system of mice with experimental allergic encephalomyelitis. Regulation by Th1 cytokines. J. Immunol. 154:944-953.

8. Merrill, J.E., et al. 1992. Inflammatory leukocytes and cytokines in the peptide-induced disease of experimental allergic encephalomyelitis in SJL and B10.PL mice. Proc. Natl. Acad. Sci. U. S. A. 89:574-578.

9. Ben Nun, A., Wekerle, H., and Cohen, I.R. 1981. The rapid isolation of clonable antigen-specific T lymphocyte lines capable of mediating autoimmune encephalomyelitis. Eur. J. Immunol. 11:195-199.

10. Pettinelli, C.B., and McFarlin, D.E. 1981. Adoptive transfer of experimental allergic encephalomyelitis in SJL/J mice after in vitro activation of lymph node cells by myelin basic protein: requirement for Lyt 1+ 2- T lymphocytes. J. Immunol. 127:1420-1423.

11. Ferber, I.A., et al. 1996. Mice with a disrupted IFN-gamma gene are susceptible to the induction of experimental autoimmune encephalomyelitis (EAE). J. Immunol. 156:5-7.

12. Frei, K., et al. 1997. Tumor necrosis factor alpha and lymphotoxin alpha are not required for induction of acute experimental autoimmune encephalomyelitis. J. Exp. Med. 185:2177-2182.

13. Willenborg, D.O., Fordham, S., Bernard, C.C., Cowden, W.B., and Ramshaw, I.A. 1996. IFNgamma plays a critical down-regulatory role in the induction and effector phase of myelin oligodendrocyte glycoprotein-induced autoimmune encephalomyelitis. J. Immunol. 157:3223-3227.

14. Liblau, R., Steinman, L., and Brocke, S. 1997. Experimental autoimmune encephalomyelitis in
IL-4-deficient mice. Int. Immunol. 9:799-803.

15. Szabo, S.J., et al. 2000. A novel transcription factor, T-bet, directs Th1 lineage commitment. Cell. 100:655-669.

16. Szabo, S.J., et al. 2002. Distinct effects of T-bet in TH1 lineage commitment and IFN-gamma production in CD4 and CD8 T cells. Science. 295:338-342.

17. Bettelli, E., et al. 2004. Loss of T-bet, but not STAT1, prevents the development of experimental autoimmune encephalomyelitis. J. Exp. Med. 200:79-87.

18. Nath, N., Prasad, R., Giri, S., Singh, A.K., and Singh, I. 2006. T-bet is essential for the progression of experimental autoimmune encephalomyelitis. Immunology. 118:384-391.

19. Neurath, M.F., et al. 2002. The transcription factor T-bet regulates mucosal $\mathrm{T}$ cell activation in experimental colitis and Crohn's disease. J. Exp. Med. 195:1129-1143.

20. Peng, S.L., Szabo, S.J., and Glimcher, L.H. 2002. T-bet regulates IgG class switching and pathogenic autoantibody production. Proc. Natl. Acad. Sci.U.S. A. 99:5545-5550.

21. Finotto, S., et al. 2001. Treatment of allergic airway inflammation and hyperresponsiveness by antisense-induced local blockade of GATA-3 expression. J. Exp. Med. 193:1247-1260.

22. Frisullo, G., et al. 2006. pSTAT1, pSTAT3, and $\mathrm{T}$-bet expression in peripheral blood mononuclear cells from relapsing-remitting multiple sclerosis patients correlates with disease activity. J. Neurosci. Res. 84:1027-1036.

23. Nakamura, Y., et al. 1999. Gene expression of the GATA-3 transcription factor is increased in atopic asthma. J. Allergy Clin. Immunol. 103:215-222.

24. Finotto, S., et al. 2002. Development of spontaneous airway changes consistent with human asthma in mice lacking T-bet. Science. 295:336-338

25. Nicholson, L.B., and Kuchroo, V.K. 1996. Manipulation of the Th1/Th2 balance in autoimmune disease. Curr. Opin. Immunol. 8:837-842.

26. Leonard, J.P., Waldburger, K.E., and Goldman, S.J. 1995. Prevention of experimental autoimmune encephalomyelitis by antibodies against interleukin 12. J. Exp. Med. 181:381-386.

27. Malfait, A.M., et al. 1998. Blockade of IL-12 during the induction of collagen-induced arthritis (CIA) markedly attenuates the severity of the arthritis. Clin. Exp. Immunol. 111:377-383.

28. Segal, B.M., Dwyer, B.K., and Shevach, E.M. 1998. An interleukin (IL)-10/IL-12 immunoregulatory circuit controls susceptibility to autoimmune disease. J. Exp. Med. 187:537-546.

29. Germann, T., Hess, H., Szeliga, J., and Rude, E. 1996. Characterization of the adjuvant effect of IL-12 and efficacy of IL-12 inhibitors in type II collagen-induced arthritis. Ann. N. Y. Acad. Sci. 795:227-240.

30. Nicoletti, F., et al. 1996. Elevated serum levels of interleukin-12 in chronic progressive multiple sclerosis. J. Neuroimmunol. 70:87-90.

31. Drulovic, J., et al. 1997. Interleukin-12 and tumor necrosis factor-alpha levels in cerebrospinal fluid of multiple sclerosis patients. J. Neurol. Sci. 147:145-150

32. Comabella, M., et al. 1998. Elevated interleukin-12 in progressive multiple sclerosis correlates with disease activity and is normalized by pulse cyclophosphamide therapy. J. Clin. Invest. 102:671-678.

33. Boxel-Dezaire, A.H., et al. 1999. Decreased interleukin-10 and increased interleukin-12p40 mRNA are associated with disease activity and characterize different disease stages in multiple sclerosis. Ann. Neurol. 45:695-703.

34. Morita, Y., et al. 1998. Expression of interleukin-12 in synovial tissue from patients with rheumatoid arthritis. Arthritis Rheum. 41:306-314.

35. Sakkas, L.I., Johanson, N.A., Scanzello, C.R., and Platsoucas, C.D. 1998. Interleukin-12 is expressed by infiltrating macrophages and synovial lining cells in rheumatoid arthritis and osteoarthritis. Cell Immunol. 188:105-110.

36. Oppmann, B., et al. 2000. Novel p19 protein engages IL-12p40 to form a cytokine, IL-23, with biological activities similar as well as distinct from IL-12. Immunity. 13:715-725.

37. Ling, P., et al. 1995. Human IL-12 p40 homodimer binds to the IL-12 receptor but does not mediate biologic activity. J. Immunol. 154:116-127.

38. Wiekowski, M.T., et al. 2001. Ubiquitous transgenic expression of the IL-23 subunit p19 induces multiorgan inflammation, runting, infertility, and premature death. J. Immunol. 166:7563-7570.

39. Becher, B., Durell, B.G., and Noelle, R.J. 2002. Experimental autoimmune encephalitis and inflammation in the absence of IL-12. J. Clin. Invest. 110:493-497. doi:10.1172/JCI200215751.

40. Gran, B., et al. 2002. IL-12p35-deficient mice are susceptible to experimental autoimmune encephalomyelitis: evidence for redundancy in the IL-12 system in the induction of central nervous system autoimmune demyelination. J. Immunol. 169:7104-7110.

41. Zhang, G.X., et al. 2003. Induction of experimental autoimmune encephalomyelitis in IL-12 receptor-beta 2-deficient mice: IL-12 responsiveness is not required in the pathogenesis of inflammatory demyelination in the central nervous system. J. Immunol. 170:2153-2160.

42. Cua, D.J., et al. 2003. Interleukin-23 rather than interleukin-12 is the critical cytokine for autoimmune inflammation of the brain. Nature. 421:744-748.

43. Murphy, C.A., et al. 2003. Divergent pro- and antiinflammatory roles for IL-23 and IL-12 in joint autoimmune inflammation. J. Exp. Med. 198:1951-1957.

44. Langrish, C.L., et al. 2005. IL-23 drives a pathogenic $T$ cell population that induces autoimmune inflammation. J. Exp. Med. 201:233-240.

45. Kennedy, J., et al. 1996. Mouse IL-17: a cytokine preferentially expressed by alpha beta TCR + CD4CD8-T cells. J. Interferon Cytokine Res. 16:611-617. 
46. Fossiez, F., et al. 1996. T cell interleukin-17 induces stromal cells to produce proinflammatory and hematopoietic cytokines. J. Exp. Med. 183:2593-2603.

47. Jovanovic, D.V., et al. 1998. IL-17 stimulates the production and expression of proinflammatory cytokines, IL-beta and TNF-alpha, by human macrophages. J. Immunol. 160:3513-3521.

48. Laan, M., et al. 1999. Neutrophil recruitment by human IL-17 via C-X-C chemokine release in the airways. J. Immunol. 162:2347-2352.

49. Aggarwal, S., and Gurney, A.L. 2002. IL-17: prototype member of an emerging cytokine family. J. Leukoc. Biol. 71:1-8.

50. Kolls, J.K., and Linden, A. 2004. Interleukin-17 family members and inflammation. Immunity. 21:467-476.

51. Gaffen, S.L., Kramer, J.M., Yu, J.J., and Shen, F. 2006. The IL-17 cytokine family. Vitam. Horm. 74:255-282.

52. Infante-Duarte, C., Horton, H.F., Byrne, M.C., and Kamradt, T. 2000. Microbial lipopeptides induce the production of IL-17 in Th cells. J. Immunol. 165:6107-6115.

53. Liang, S.C., et al. 2006. Interleukin (IL)-22 and IL-17 are coexpressed by Th17 cells and cooperatively enhance expression of antimicrobial peptides. J. Exp. Med. 203:2271-2279.

54. Chung, Y., et al. 2006. Expression and regulation of IL-22 in the IL-17-producing CD4+ T lymphocytes. Cell Res. 16:902-907.

55. Zheng, Y., et al. 2006. Interleukin-22, a T(H)17 cytokine, mediates IL-23-induced dermal inflammation and acanthosis. Nature. 445:648-651.

56. Chabaud, M., et al. 1999. Human interleukin-17: a $\mathrm{T}$ cell-derived proinflammatory cytokine produced by the rheumatoid synovium. Arthritis Rheum. 42:963-970.

57. Kotake, S., et al. 1999. IL-17 in synovial fluids from patients with rheumatoid arthritis is a potent stimulator of osteoclastogenesis. J. Clin. Invest. 103:1345-1352.

58. Matusevicius, D., et al. 1999. Interleukin-17 mRNA expression in blood and CSF mononuclear cells is augmented in multiple sclerosis. Mult. Scler. 5:101-104.

59. Lock, C., et al. 2002. Gene-microarray analysis of multiple sclerosis lesions yields new targets validated in autoimmune encephalomyelitis. Nat. Med. 8:500-508

60. Komiyama, Y., et al. 2006. IL-17 plays an important role in the development of experimental autoimmune encephalomyelitis. J. Immunol. 177:566-573.

61. Nakae, S., Nambu, A., Sudo, K., and Iwakura, Y. 2003. Suppression of immune induction of collagen-induced arthritis in IL-17-deficient mice. J. Immunol. 171:6173-6177.

62. Bush, K.A., Farmer, K.M., Walker, J.S., and Kirkham, B.W. 2002. Reduction of joint inflammation and bone erosion in rat adjuvant arthritis by treatment with interleukin-17 receptor IgG1 Fc fusion protein. Arthritis Rheum. 46:802-805.

63. Park, H., et al. 2005. A distinct lineage of CD4 T cells regulates tissue inflammation by producing interleukin 17. Nat. Immunol. 6:1133-1141.

64. Veldhoen, M., Hocking, R.J., Atkins, C.J., Locksley, R.M., and Stockinger, B. 2006. TGFbeta in the context of an inflammatory cytokine milieu supports de novo differentiation of IL-17-producing T cells. Immunity. 24:179-189.

65. Bettelli, E., et al. 2006. Reciprocal developmental pathways for the generation of pathogenic effector TH17 and regulatory T cells. Nature. 441:235-238.

66. Ivanov, I.I., et al. 2006. The orphan nuclear receptor RORgammat directs the differentiation program of proinflammatory IL-17+ T helper cells. Cell. 126:1121-1133.

67. Harrington, L.E., et al. 2005. Interleukin 17-producing CD4+ effector $\mathrm{T}$ cells develop via a lineage distinct from the T helper type 1 and 2 lineages. Nat. Immunol. 6:1123-1132.

68. Chen, Y., et al. 2006. Anti-IL-23 therapy inhibits multiple inflammatory pathways and ameliorates autoimmune encephalomyelitis. J. Clin. Invest. 116:1317-1326. doi:10.1172/JCI25308.

69. Becher, B., Durell, B.G., and Noelle, R.J. 2003. IL-23 produced by CNS-resident cells controls T cell encephalitogenicity during the effector phase of experimental autoimmune encephalomyelitis. J. Clin. Invest. 112:1186-1191. doi:10.1172/ JCI200319079.

70. McIntyre, K.W., et al. 1996. Reduced incidence and severity of collagen-induced arthritis in interleukin12-deficient mice. Eur. J. Immunol. 26:2933-2938.

71. Shi, F.D., Takeda, K., Akira, S., Sarvetnick, N., and Ljunggren, H.G. 2000. IL-18 directs autoreactive T cells and promotes autodestruction in the central nervous system via induction of IFN-gamma by NK cells. J. Immunol. 165:3099-3104

72. Wei, X.Q., Leung, B.P., Arthur, H.M., McInnes, I.B., and Liew, F.Y. 2001. Reduced incidence and severity of collagen-induced arthritis in mice lacking IL-18. J. Immunol. 166:517-521.

73. Leung, B.P., McInnes, I.B., Esfandiari, E., Wei, X.Q., and Liew, F.Y. 2000. Combined effects of IL-12 and IL-18 on the induction of collagen-induced arthritis. J. Immunol. 164:6495-6502.

74. Canetti, C.A., et al. 2003. IL-18 enhances collagen-induced arthritis by recruiting neutrophils via TNF-alpha and leukotriene B4. J. Immunol. 171:1009-1015.

75. Horwood, N.J., et al. 1998. Interleukin 18 inhibits osteoclast formation via $\mathrm{T}$ cell production of granulocyte macrophage colony-stimulating factor. J. Clin. Invest. 101:595-603.

76. Udagawa, N., et al. 1997. Interleukin-18 (interferon-gamma-inducing factor) is produced by osteoblasts and acts via granulocyte/macrophage colony-stimulating factor and not via interferongamma to inhibit osteoclast formation. J. Exp. Med. 185:1005-1012.

77. Dai, S.M., Nishioka, K., and Yudoh, K. 2004. Interleukin (IL) 18 stimulates osteoclast formation through synovial $\mathrm{T}$ cells in rheumatoid arthritis: comparison with IL1 beta and tumour necrosis factor alpha. Ann. Rheum. Dis. 63:1379-1386.

78. Sato, K., et al. 2006. Th17 functions as an osteoclastogenic helper $\mathrm{T}$ cell subset that links $\mathrm{T}$ cell activation and bone destruction. J. Exp. Med. 203:2673-2682.

79. Gutcher, I., Urich, E., Wolter, K., Prinz, M., and Becher, B. 2006. Interleukin 18-independent engagement of interleukin 18 receptor-alpha is required for autoimmune inflammation. Nat. Immunol. 7:946-953.

80. Santos, L.L., et al. 2006. IL-18 is redundant in T-cell responses and in joint inflammation in antigeninduced arthritis. Immunol. Cell Biol. 84:166-173.

81. Jiang, H.R., et al. 2001. IL-18 not required for IRBP peptide-induced EAU: studies in gene-deficient mice. Invest. Ophthalmol. Vis. Sci. 42:177-182.

82. Torigoe, K., et al. 1997. Purification and characterization of the human interleukin-18 receptor. J. Biol. Chem. 272:25737-25742.

83. Lewis, E.C., and Dinarello, C.A. 2006. Responses of IL-18- and IL-18 receptor-deficient pancreatic islets with convergence of positive and negative signals for the IL-18 receptor. Proc. Natl. Acad. Sci. U. S. A. 103:16852-16857.

84. Bailey, S.L., Schreiner, B., McMahon, E.J., and Miller, S.D. 2007. CNS myeloid DCs presenting endogenous myelin peptides 'preferentially' polarize CD4(+) T(H)-17 cells in relapsing EAE. Nat. Immunol. 8:172-180.

85. Fontana, A., et al. 1982. Interleukin 1 activity in the synovial fluid of patients with rheumatoid arthritis. Rheumatol. Int. 2:49-53.
86. Hirano, T., et al. 1988. Excessive production of interleukin $6 / \mathrm{B}$ cell stimulatory factor- 2 in rheumatoid arthritis. Eur. J. Immunol. 18:1797-1801.

87. Navikas, V., et al. 1996. Increased interleukin-6 mRNA expression in blood and cerebrospinal fluid mononuclear cells in multiple sclerosis. J. Neuroimmunol. 64:63-69.

88. Okuda, Y., et al. 1998. IL-6-deficient mice are resistant to the induction of experimental autoimmune encephalomyelitis provoked by myelin oligodendrocyte glycoprotein. Int. Immunol. 10:703-708.

89. Alonzi, T., et al. 1998. Interleukin 6 is required for the development of collagen-induced arthritis. J. Exp. Med. 187:461-468.

90. Saijo, S., Asano, M., Horai, R., Yamamoto, H., and Iwakura, Y. 2002. Suppression of autoimmune arthritis in interleukin-1-deficient mice in which T cell activation is impaired due to low levels of CD40 ligand and OX40 expression on T cells. Arthritis Rheum. 46:533-544.

91. Schiffenbauer, J., et al. 2000. The induction of EAE is only partially dependent on TNF receptor signaling but requires the IL-1 type I receptor. Clin. Immunol. 95:117-123.

92. Sutton, C., Brereton, C., Keogh, B., Mills, K.H., and Lavelle, E.C. 2006. A crucial role for interleukin (IL)- 1 in the induction of IL-17-producing T cells that mediate autoimmune encephalomyelitis. J. Exp. Med. 203:1685-1691.

93. Nakae, S., et al. IL-17 production from activated T cells is required for the spontaneous development of destructive arthritis in mice deficient in IL-1 receptor antagonist. Proc. Natl. Acad. Sci. U. S. A. 100:5986-5990.

94. Hirota, K., et al. 2007. T cell self-reactivity forms a cytokine milieu for spontaneous development of IL-17+ Th cells that cause autoimmune arthritis. J. Exp. Med. 204:41-47.

95. Kleinschek, M.A., et al. 2007. IL-25 regulates Th17 function in autoimmune inflammation. J. Exp. Med. 204:161-170.

96. Underhill, G.H., et al. 2005. A crucial role for T-bet in selectin ligand expression in T helper 1 (Th1) cells. Blood. 106:3867-3873.

97. Lord, G.M., et al. 2005. T-bet is required for optimal proinflammatory CD4+ T-cell trafficking. Blood. 106:3432-3439.

98. Drulovic, J., et al. 1998. Serum interleukin-12 levels in patients with multiple sclerosis. Neurosci. Lett. 251:129-132.

99. Windhagen, A., et al. 1995. Expression of costimulatory molecules B7-1 (CD80), B7-2 (CD86), and interleukin 12 cytokine in multiple sclerosis lesions. J. Exp. Med. 182:1985-1996.

100.Clerici, M., et al. 2001. Single-cell analysis of cytokine production shows different immune profiles in multiple sclerosis patients with active or quiescent disease. J. Neuroimmunol. 121:88-101.

101. Ozenci, V., et al. 2001. IL-12/IL-12R system in multiple sclerosis. J. Neuroimmunol. 114:242-252.

102.Bucht, A., et al. 1996. Expression of interferongamma (IFN-gamma), IL-10, IL-12 and transforming growth factor-beta (TGF-beta) mRNA in synovial fluid cells from patients in the early and late phases of rheumatoid arthritis (RA). Clin. Exp. Immunol. 103:357-367.

103. Nicoletti, F., et al. 2001. Increased serum levels of interleukin-18 in patients with multiple sclerosis. Neurology. 57:342-344.

104.Balashov, K.E., Rottman, J.B., Weiner, H.L., and Hancock, W.W. 1999. CCR5(+) and CXCR3(+) T cells are increased in multiple sclerosis and their ligands MIP-1alpha and IP-10 are expressed in demyelinating brain lesions. Proc. Natl. Acad. Sci. U. S. A. 96:6873-6878.

105.Huang, W.X., Huang, P., and Hillert, J. 2004. Increased expression of caspase- 1 and interleukin18 in peripheral blood mononuclear cells in patients 
with multiple sclerosis. Mult. Scler. 10:482-487.

106. Gracie, J.A., et al. 1999. A proinflammatory role for IL-18 in rheumatoid arthritis. J. Clin. Invest. 104:1393-1401.

107. Tanaka, M., et al. 2001. Mature form of interleukin 18 is expressed in rheumatoid arthritis synovial tissue and contributes to interferon-gamma production by synovial T cells. J. Rheumatol. 28:1779-1787.

108. Rooney, T., et al. 2004. Synovial tissue interleukin-

18 expression and the response to treatment in patients with inflammatory arthritis. Ann. Rheum. Dis. 63:1393-1398.

109.Dettke, M., Scheidt, P., Prange, H., and Kirchner,
H. 1997. Correlation between interferon production and clinical disease activity in patients with multiple sclerosis. J. Clin. Immunol. 17:293-300.

110.Petereit, H.F., Richter, N., Pukrop, R., and Bamborschke, S. 2000. Interferon gamma production in blood lymphocytes correlates with disability score in multiple sclerosis patients. Mult. Scler. 6:19-23.

111.Wildbaum, G., Youssef, S., Grabie, N., and Karin, N. 1998. Neutralizing antibodies to IFN-gammainducing factor prevent experimental autoimmune encephalomyelitis. J. Immunol. 161:6368-6374.

112.Plater-Zyberk, C., et al. 2001. Therapeutic effect of neutralizing endogenous IL-18 activity in the collagen-induced model of arthritis. J. Clin. Invest. 108:1825-1832. doi:10.1172/JCI200112097.

113. Chu, C.Q., Song, Z., Mayton, L., Wu, B., and Wooley, P.H. 2003. IFNgamma deficient C57BL/6 (H-2b) mice develop collagen induced arthritis with predominant usage of T cell receptor Vbeta6 and Vbeta8 in arthritic joints. Ann. Rheum. Dis. 62:983-990.

114. Manoury-Schwartz, B., et al. 1997. High susceptibility to collagen-induced arthritis in mice lacking IFN-gamma receptors. J. Immunol. 158:5501-5506.

115.Vermeire, K., et al. 1997. Accelerated collageninduced arthritis in IFN-gamma receptor-deficient mice. J. Immunol. 158:5507-5513. 